\title{
2 Ausblick
}

\subsection{Implikationen für die Designtheorie und -praxis}

Die Rhetorik hat sich als fruchtbare Heuristik erwiesen, um Zusammenhänge der Elaboration und Imperfektion sowie des Dilettantismus für das Design besser verstehen zu können. Sowohl zu den Elaborationsidealen als auch zu den Strategien der Imperfektion und dem Reiz des Dilettantischen lassen sich rhetorische Ansätze finden, welche auf das Design übertragen werden können. Auf verschiedenen Ebenen könnten Themen und Erkenntnisse dieser Arbeit noch weiter ausgeführt werden.

Auf einer übergeordneten Systemebene ließen sich die fast schon zyklischen Veränderungen, Brüche, und Verschiebungen in den Téchnai der Rhetorik und des Designs, die sich in dieser Arbeit angedeutet haben, weiterverfolgen. Zum einen könnte überprüft werden, ob sich das beobachtete Wechselspiel von Erneuerung, Konventionalisierung und Konsolidierung der kommunikativen und gestalterischen Elaborationstechniken, von Regelbefolgung und Regelbruch, die wieder zu neuen Perfektions- und Elaborationsidealen führen, im Hinblick auf weitere Strömungen und Gegenbewegungen in Design, Kunst und Populärkultur anwenden lässt. So könnten etwa die in raschem Wandel begriffenen Authentizitätstechniken in den Social Media aus diesem Blickwinkel untersucht werden. Zum anderen könnten die angestellten Beobachtungen durch eine theoretische, insbesondere sozialwissenschaftliche Vertiefung von Begriffen wie 〈Regelbruch〉 oder «Systemwechsel〉 noch besser fundiert werden. Hierfür könnte beispielsweise Thomas Kuhns Modell des «Paradigmenwechsels` in der Wissenschaft hilfreich sein (Kuhn 1962). Es wäre zu untersuchen, ob bei den einzelnen Brüchen neue Paradigmen bzw. radikal andere Denkweisen eingeführt werden oder ob es hier lediglich um Fokusverschiebungen innerhalb eines Systems geht. Um das in dieser Arbeit skizzierte zyklische bzw. dynamische Gestaltungsmodell weiter auszuarbeiten und die Rede von 〈Systemen〉 zu fundieren, könnte die Systemtheorie konsultiert oder in Richtung der 〈Actor Network Theory〉 (Latour 2005) weitergedacht werden, in welcher visuelle Kommunikationsmittel bzw. Designartefakte selbst als Akteure innerhalb des Netzwerks zu konzipieren sind.

Konkret ergeben sich aus der Berücksichtigung der Alltags- und Laiengestaltung und anderer Formen wenig elaborierter Gestaltung neue Betrachtungsund Evaluationsweisen für die Designtheorie und -ausbildung. Bis heute ist die Aufmerksamkeit meist auf die Ränder und Bruchstellen der Gestaltung gerichtet. Experimentelles, Herausragendes und Abweichendes erhält Beachtung und Anerkennung. Konventionelles, Durchschnittliches und Triviales dagegen fällt 
durch die Maschen. Dabei gibt es zum einen, wie diese Studie nahelegt, durchaus eine Interaktion zwischen dem «Mainstream〉 der Gestaltung und den herausragenden, meisterhaften Werken, die sich davon abheben. Erst durch die Verbreitung und Anpassung an den Massengeschmack und den damit einhergehenden Überdruss entsteht ein Ansporn und ein Sprungbrett für neue Abweichungsmodi. Zum andern beziehen sich die Motoren der Erneuerung vielfach auf gestalterische Alltags- und Laienaktivitäten, welche vom Radar des Regelwerks noch nicht erfasst wurden: «unverfälschte〉, ungeschliffene visuelle Formen, welche zur Erfrischung und Erneuerung der professionellen Gestaltungspraxis beitragen können. Ein vertieftes Verständnis gestalterischer Laienpraktiken und der populären Alltagskultur wäre somit für ein umfassendes Verständnis auch der professionellen Design- und Evaluationspraxis unabdingbar.

Um die gesellschaftliche Dimension, die Relevanz und den Status des Phänomens «Amateurgestaltung〉 umfassender zu ermitteln, könnten Pierre Bourdieus und Luc Boltanskis soziologischen Studien zur Fotografie als einer profanen Tätigkeit oder «Volkskunst» (Bourdieu 1981 [1965]: 19) herangezogen werden (beide in Bourdieu et al. 1965). Denn jene «Demokratisierung〉 der Produktionsmittel, welche sich heute im Grafikdesign feststellen lässt, vollzog sich in der Fotografie bereits im Verlauf des 20. Jahrhunderts:

Da die Beschäftigung mit der Photographie - im Gegensatz zu den künstlerischen Tätigkeiten, die, wie die Malerei oder die Musik, etwas ganz und gar Weihevolles an sich haben technisch wie ökonomisch jedermann zugänglich erscheint, und da diejenigen, die sich ihr widmen, von sich selbst nicht das Gefühl haben, an einem System expliziter und kodifizierter Normen gemessen zu werden, das die legitime Praxis im Hinblick auf ihren Gegenstand, ihre Anlässe und ihre Modalität festlegte, ist die Analyse der subjektiven oder objektiven Bedeutung, die die Subjekte der Photographie als Praxis oder als kultureller Tätigkeit beimessen, besonders geeignet, an deren authentischer Ausdrucksform jene Ästhetiken (und Ethiken) zu ermitteln, die für unterschiedliche Gruppen oder Klassen kennzeichnend sind, vor allem die «Ästhetik〉 des «einfachen Volkes〉, die sich hier in Ausnahmefällen offenbaren kann. (Bourdieu 1981 [1965]: 18; Hervorhebungen A.S.)

Gleichzeitig weist Bourdieu darauf hin, dass die Amateurpraktiken des Fotografierens nur den Anschein wecken, «ohne Traditionen und ohne Ansprüche, gänzlich anarchisch, individuelle Improvisation» (Bourdieu 1981 [1965]: 18) zu sein, dabei jedoch bereits selbst einer starken, oft impliziten Reglementierung unterworfen sind - was sich in den stereotypen Motiven und gestellten Kompositionen von Amateurfotos etwa auf Urlaubsbildern zeige. Stark standardisierte, fast schon austauschbare Elaborationstypen der gestalterischen Laienproduktion konnten wir in Form der einfachen «Wordgestaltung〉 in der Gemeinwesenarbeit ebenfalls kennenlernen. Bourdieu kritisiert zudem, dass diese «durchschnitt- 
lichen> Praktiken und die daraus resultierenden ästhetischen Formen keine Beachtung und Legitimierung als Kunst erhielten: «Kurz, es ist möglich, daß der größte Teil der Gesellschaft aus dem Universum der legitimen Kultur ausgeschlossen bleibt, ohne deshalb aus dem Universum des Ästhetischen verbannt zu sein.» (Bourdieu 1981 [1965]: 19). Beide Beobachtungen sind insofern relevant für die in dieser Arbeit untersuchten Zusammenhänge zwischen den Wirkungsweisen einer perfekten Ausarbeitung und der strategischen oder dilettantischen Imperfektion, als sich professionelle Fotografen wiederum jener dilettantisch-fehlerhaften Schnappschuss-Ästhetik bedienen können, um Authentizität zu suggerieren - selbst dann, wenn nicht einmal die Laienaktivität in der Realität komplett regellos, «natürlich〉 oder naiv abläuft. Genau dieses «rhetorische〉 Zusammenspiel zwischen der vermeintlich spontan und unschuldig auftretenden technischen Imperfektion und der damit verfolgten Wirkungsintention führt Boltanski für die Fotografie am Beispiel der «Unschärfe» weiter aus:

Nicht nur, dass sich die Photographie des Ereignisses, wie es sich gerade vollzieht, unscharfe Konturen leisten kann, die Unschärfe macht geradezu ihr Charakteristikum aus. Die Unschärfe ist ein Vehikel, um den Betrachter davon zu überzeugen, daß das Bild tatsächlich das Ereignis selbst zeigt und in dem Augenblick gemacht wurde, als es sich abspielte, und zwar auf mechanische Weise und - damit ist alles gesagt - objektiv. So ist die Unschärfe, die im allgemeinen Bewußtsein gleichbedeutend ist mit Bewegung, der beste Ausweis für die Reinheit der Absichten des Photographen. Die Überzeichnung der Kontraste, der ungeschickte Bildausschnitt, das grobe Korn, all dies verweist auf den Moment, in dem das Bild eingefangen wurde, und läßt sich als Indiz der Schwierigkeiten lesen, die im Augenblick der Aufnahme zu bewältigen waren. (Boltanski 1981 [1965]: 158-159; Hervorhebungen A.S.)

Es zeigt sich hier noch einmal im Kern, dass Aspekte der bewusst niedrigen, fehlerhaften, nachlässigen oder dilettantischen Elaboration zu einem zentralen Wirkfaktor der visuellen Bild- und Gestaltungsproduktion werden können. Es ist frappant, wie die beschriebenen fotografischen Praktiken den schon in der klassischen Rhetorik beschriebenen Techniken der Dissimulatio Artis und des Suggerierens von Natürlichkeit, Aufrichtigkeit, Unmittelbarkeit, Spontaneität und Nonchalance gleichen, die heute unter dem Begriff der Authentizität zusammengefasst werden.

Das potenzielle Anwachsen der semiprofessionellen Gestaltungsarbeit durch «professionelle Amateure ist nicht nur ein designtheoretisch und -historisch unaufgearbeitetes Thema, sondern könnte in Zukunft zu einer existenziellen Herausforderung für die professionelle Gestaltungspraxis werden. Das Material allerdings, das in der vorliegenden Arbeit untersucht wurde, erreicht bei weitem noch kein Elaborationsniveau, das zu einer ernsthaften Bedrohung für die Profession werden könnte. Die analysierten semiprofessionellen Artefakte fallen durch man- 
gelhafte gestalterische Qualität, visuelle Unbestimmtheit oder Überladenheit auf. Aus Sicht der Experten war die Anzahl von Artefakten im semiprofessionellen Grenzbereich noch erstaunlich klein. Mit dem Heranwachsen der «Digital Natives` könnte hier ein Generationenwechsel anstehen. Welche Probleme daraus für das Berufsfeld erwachsen und welche Qualifikationen professionelle Grafikdesigner in Zukunft brauchen werden, um sich von der semiprofessionellen Tätigkeit abzuheben, müsste zum Gegenstand weiterer Forschung werden, um eine rechtzeitige Anpassung des Berufsbilds und der Ausbildung im Design einzuleiten.

Gestalterische Mittelmäßigkeit und der «konventionelle» professionelle Elaborationstyp sind verbreitet in den beiden untersuchten Gebieten Gemeinwesenarbeit und Lokalpolitik. Besonders in der lokalpolitischen Kommunikation wird ein gewisser Konformismus nicht nur toleriert, sondern er scheint gar unumstößlichen Gesetzen der Massentauglichkeit und Wiedererkennbarkeit geschuldet: «Das Publikum bekommt, was es verdient». Ein Befund aus dem durchgeführten Designexperiment lässt jedoch hellhörig werden, da er auf eine mögliche Diskrepanz zwischen Selbst- und Fremdwahrnehumg des Zielpublikums hindeutet: Während die höher gebildeten Testpersonen vermuteten, dass der konventionell und mittelmäßig gestaltete Wahlprospekt der BDP beim 〈Volk〉 am besten ankomme, erachteten jene Personen, welche sich politisch in der Nähe der Absenderpartei sehen und somit das Zielpublikum bilden, meist den hoch elaborierten Prospekt für den angemessensten. Hat also das «gemeine Volk〉 durchaus einen Sinn für gestalterische Qualität (analog zu Ciceros Sensus naturalis in sprachlichen Belangen), der ihm jedoch von den «Eliten` abgesprochen wird? Angesichts der beschriebenen Diskrepanz stellt sich die Frage, was «Angemessenheit hier bedeutet bzw. wer die Entscheidungsmacht über die «angemessene> Lösung hat - was sich weiter zu untersuchen lohnt. Das Aptum erweist sich als der wesentlich komplexere Elaborationsfaktor als die gestalterische Qualität und Könnerschaft: Über den Professionalitätsgrad gestalterischer Artefakte sind sich Experten- und Laienpublikum sowie gebildete und weniger gebildete Personen erstaunlich einig.

Ein weiterer Faktor, der in der Designforschung noch wenig Beachtung gefunden hat, ist der Zusammenhang von Gestaltung und Geld. Was soll und darf die visuelle Kommunikation kosten? Wie in der Designstudie aufgezeigt werden konnte, werden die verfügbaren sowie eingesetzten Geldmittel sowohl in der Lokalpolitik als auch in der Gemeinwesenarbeit rhetorisch wirksam: Die Kommunikationsbudgets der Gemeinwesenarbeit sind knapp und es kann und soll in diesem Bereich nicht um Werbung und PR, sondern um die gemeinnützige Arbeit gehen. Diese Restriktion sollte sich auch im Elaborationsgrad der visuellen Kommunikation niederschlagen - was mit «selbstgemachter» Wordgestaltung ganz gut gelingt. In der Politik halten die Stimmberechtigten zu Recht ein Auge 
auf die für den Wahlkampf eingesetzten Mittel: Geld kann den demokratischen Prozess korrumpieren. Aufwändig produzierte Kommunikationsmittel zeigen an, dass entsprechende Geldmittel vorhanden sind und für die Kommunikation aufgewendet wurden. Selbstgemachte, einfache Grafik dagegen weist implizit auf ihre billige Produktionsweise hin. Sowohl für die Gemeinwesenarbeit als auch für die Lokalpolitik kann sich wenig elaborierte und somit auch billigere Gestaltung positiv auswirken: Dass das Geld nicht für die Bewerbung einer Sache, sondern für die Sache selbst gebraucht wird, hat sich in den Expertenanalysen und den Befragungen des Laienpublikums als wichtiges Argument erwiesen. Wo wenig Geld im Spiel ist, darauf hat schon Quintilian aufmerksam gemacht, empfiehlt sich eine einfache Sprache, welche die Sorgfalt verbirgt. Im Design ist die Verbindung zwischen einem hohen Elaborationsgrad und Geld noch viel deutlicher - denn ein fundiertes, professionelles Gestaltungskonzept und eine qualitativ hochwertige technische Produktion generieren Kosten. «Understatement〉 in finanziellen Belangen kann also ein weiterer Grund sein, gestalterische «Höchstleistungen〉 zu vermeiden. Dennoch ist aus Sicht der Gestaltungsexperten die angetroffene Fülle an gestalterischem Mittelmaß und allzu billig wirkender Papierqualität gerade angesichts der teils beträchtlichen Ausgaben, die größere Parteien für Kommunikation tätigen, ernüchternd.

Besonders für die visuelle Kommunikation in der Gemeinwesenarbeit, aber auch bei Kleinparteien sind limitierte Ausgaben nicht nur als bewusst gewähltes Wirkungsmittel, sondern als eine reale Restriktion zu betrachten. Wofür sollen die beschränkten Geldmittel ausgegeben werden? Lohnt es sich, die Mittel zu bündeln, um wenige Kommunikationsmittel professionell zu gestalten? Sind professionelle 〈Templates〉 für die Weiterbearbeitung durch Laien die Lösung? Würde sich eine Schulung der Laiengestalter in den Institutionen und Parteisekretariaten anbieten? Oder reicht der Status quo der Amateurgestaltung aus? Noch scheint besonders im Bereich der Gemeinwesenarbeit der «unkomplizierte` Elaborationstyp der Laiengestaltung «außer Konkurrenz» zu laufen - er muss sich nicht an «normalen` Maßstäben der gestalterischen Qualität bemessen, solange er lesbar und verständlich ist. Dennoch wäre hier zu fragen, ob nicht zumindest jene Institutionen des Gemeinwesens, die Mitglieder verlieren oder in denen Generationenwechsel anstehen, etwas mehr Sorgfalt, Emotionalität und Plakativität oder eben auch Geldmittel in die professionelle visuelle Kommunikation investieren sollten. Gerade 〈Selbstläufer〉 wie das Kerzenziehen in der Vorweihnachtszeit oder Kinderkleiderbörsen in den Quartierzentren böten hier die Möglichkeit, auch Außenstehende und jüngere Menschen anzusprechen. Spezifisch für die zwei untersuchten Felder der grafischen Gestaltung sollen abschließend die wichtigsten Einsichten und Problemstellungen, die sich aus der praxisbasier- 
ten Designstudie ergeben haben, im Sinne einer tentativen Handlungsanweisung zusammengefasst werden.

\section{Empfehlungen für die visuelle Kommunikation der Lokalpolitik}

- Grundsätzlich sollte auf Ebene der lokalpolitischen Kommunikation professionelles Grafikdesign verwendet werden. Auch ein ländliches oder weniger gebildetes Publikum vermag gestalterische Qualität, aber auch Fehlerhaftigkeit zu erkennen.

- Je wichtiger eine Politikerin, je höher das angestrebte Amt, je etablierter eine Partei, je offizieller das Kommunikationsmittel ist, desto elaborierter und professioneller muss der visuelle Auftritt sein.

- Konventionelle Gestaltungslösungen im Sinne des klassischen Wahlprospekts, die nach dem Prinzip «Köpfe zeigen〉 funktionieren und auf dem Corporate Design der Partei basieren, sind ein zwar unorigineller, aber nach wie vor gangbarer und sicherer Weg.

- Rand- und Jungparteien können sich mit engagierten gestalterischen Eigenleistungen oder schrägen, originellen Ideen vom «Mainstream〉 abheben. Gestalterische Dilettantismen sind hier in einem gewissen Maß zulässig - ja sie verstärken sogar die wahrgenommene Authentizität.

- Wer sich durch Originalität von den anderen Bewerbern abheben will, sollte ein durchdachtes Konzept vorlegen. Halbherzige Ideen, die inhaltlich oder an der gestalterischen Umsetzung scheitern, wirken sich rasch nachteilig auf das Image und die Seriosität der Absenderpartei aus.

- Sowohl die Professionalität als auch der technische und materielle Elaborationsgrad müssen mit der politischen Botschaft der Partei übereinstimmen und ihre Auffassung und finanziellen Möglichkeiten in angemessener Weise widerspiegeln: Kein 〈Klotzen〉, aber auch kein 〈Kleckern〉 bei den Bürgerlichen, Zurückhaltung bei den Sozialdemokraten, Recycling-Optik bei den Grünen, «Handgestricktes〉 bei den Alternativen etc.

\section{Empfehlungen für die visuelle Kommunikation der Gemeinwesenarbeit}

- Amateurgestaltung übernimmt aufgrund minimaler Kommunikationsbudgets eine wichtige Funktion zur Bekanntmachung von Veranstaltungen der Institutionen des Gemeinwesens.

- Amateurgestaltung bildet einen schlichten, wiedererkennbaren Elaborationstyp mit deutlich erkennbaren Dilettantismen und Defiziten in der gestalterischen Qualität, aber auch mit positiver Ausstrahlung: Sie kann Authentizität, persönliches Engagement, Vertrautheit und Nähe vermitteln.

- Minimale Anforderungen an Sorgfalt, Lesbarkeit, Verständlichkeit, Emotionalität und Attraktivität gelten auch bei selbstgestalteten Kommunikations- 
mitteln. Selbst mit rudimentären Kenntnissen und einfachsten gestalterischen Mitteln lassen sich Aussage (Logos), Charakter (Ethos) und Stimmung (Pathos) von Absender und Anlass angemessen wiedergeben.

- Die immer stärker aufkommende semiprofessionelle Gestaltungsarbeit zeigt sich bislang noch zu undefiniert oder überfrachtet, um einen Mehrwert $\mathrm{zu}$ erbringen. Sie büßt die authentische Qualität der Laienarbeit ein, entfaltet aber (noch) nicht die Qualitäten der Profiarbeit. Sie ist weder schlicht, technisch geschliffen, plakativ, noch engagiert, sympathisch oder originell.

- Für wichtige Anlässe mit Breitenwirkung, zur Bekanntmachung im öffentlichen Raum, sowie für allgemeine oder offizielle Informationen zu den Institutionen des Gemeinwesens empfiehlt sich professionelle Gestaltungsarbeit, um genügend Aufmerksamkeit zu erlangen und die Seriosität der Absenderinstitution zu unterstreichen. Hier empfiehlt es sich, die vorhandenen Geldmittel für einen durchdachten, wiedererkennbaren und auch von Laiengestaltern innerhalb der Institution wiederverwendbaren Gestaltungsauftritt $\mathrm{zu}$ bündeln.

\subsection{Implikationen für die Literaturwissenschaft und -produktion}

Die gewonnenen Erkenntnisse über die verschiedenen Aspekte, Wirkungsweisen und Ideale der Elaboration in Rhetorik und Design, aber auch ihr Wechselspiel mit dem Imperfekten, Ungeschliffenen und Fehlerhaften lassen sich in vielfacher Weise auf literaturwissenschaftliche Belange anwenden - und können auch für die Literaturproduktion und die Verlagsarbeit fruchtbar gemacht werden. In den letzten Jahrzehnten wurde die Rhetorik wieder vermehrt als Instrumentarium zur Text- und Literaturanalyse entdeckt - von der Untersuchung der Figuralität von Texten über das Aufspüren persuasiver Muster bis hin zur Erfassung literarischer Affizierungstechniken. Zudem kommen in der Literaturwissenschaft zunehmend auch Aspekte der Materialität und Visualität literarischer Werke in den Fokus. Nur spärlich wurden dabei bis heute jedoch die «ur-rhetorischen〉 Prozesse von Elaboration und Imperfektion in den Blick genommen: das Wechselspiel von kunstvoller Ausarbeitung und gleichzeitiger Verbergung der Kunst, von Anpassung und Abweichung, von Sorgfalt und Leichtigkeit, von Glaubwürdigkeit und Unmittelbarkeit, von Eloquenz und Authentizität. Wie die erarbeiteten Zusammenhänge von Elaboration und Imperfektion auf die Literaturwissenschaft und die Produktionsbedingungen literarischer Werke oder sogar auf größere Umwälzungsprozesse in der Literaturgeschichte angewendet werden könnten, soll im Folgenden anhand von zwei möglichen Ansätzen illustriert werden. 


\subsubsection{Die kritische Grenze von Fehler und Figur}

Ebensowenig wie zwischen den eigentl. Wörtern u. den Tropen ein Unterschied ist, giebt es einen zwischen der regelrechten Rede und den sogenannten rhetorischen Figuren. Eigentlich ist alles Figuration, was man gewöhnliche Rede nennt. Die Sprache wird geschaffen von den einzelnen Sprachkünstlern, festgestellt aber dadurch daß der Geschmack der Vielen eine Auswahl trifft. [...] Eine Figur, welche keine Abnehmer findet, wird Fehler. Ein von irgend einem usus angenommener Fehler wird Figur. (Nietzsche 1995 [1874]: 427-428)

Man muss nicht, wie Nietzsche dies tut, die Unterscheidung von wörtlicher und uneigentlicher Bedeutung insgesamt in Frage stellen, um einzusehen, dass die Unterscheidung zwischen Sprachkunst und sprachlichem Fehler im Einzelfall keine objektive ist, sondern innerhalb einer Gemeinschaft, eines Diskurses, eines «Usus» entschieden wird. Das Zitat spricht nicht nur die bereits in der antiken Rhetorik manifeste Problematik an, dass Fehler und Figur eng beieinander liegen, sondern es verweist auch auf die dort beschriebene Bedeutung des Decorum, des geltenden Sprachgebrauchs und jener Auctoritates, welche über die Grenzziehung zwischen zulässiger Abweichung und Vitium entscheiden: Was sich ziemt, was einen Abnehmer findet, was den Geschmack des Publikums trifft oder was Anerkennung von der Fachwelt erhält, ist auch das Richtige - «Aber über das Maß ist großer Streit: der Eine ist da entzückt, wo der andere widrige Fehler empfindet» (Nietzsche 1995 [1874]: 428). Was im Schulaufsatz mit dem Rotstift markiert wird, kann in Werken der Literatur nicht nur toleriert werden, sondern zu einem wichtigen Elaborations-, Distinktions- und Qualitätsmerkmal eines Werks oder Autors werden: Wortneubildungen, das Einweben umgangssprachlicher Wendungen, inkonsequenter oder unvollständiger Satzbau, syntaktische Brüche, die penetrante Wiederholung von Wörtern, das Einbauen stilfremder Elemente und unzählige weitere Möglichkeiten der unelaborierten, defekten, imperfekten, von Grammatik und Sprachnorm abweichenden Sprachmerkmale. Ob diese Elaborationsweisen als Stilblüten, Fehler oder Dilettantismen zu verwerfen sind oder gerade als Qualitäten eines eigenständigen literarischen Stils geschätzt werden, entscheidet sich im heutigen Literaturbetrieb schon früh: Pauschal bei der Auswahl oder Ablehnung von Manuskripten im Verlag, im Einzelnen dann beim Lektorat der Textfassung. Sind die Werke publiziert, legen Literaturkritiker und schließlich Literaturwissenschaftler ihre jeweiligen Elaborations- und Qualitätsmaßstäbe an die Texte an. Mit zunehmender Berühmtheit einer Autorin und Kanonisierung ihres Werks wird auch die Tendenz zunehmen, die im Text wahrgenommenen Imperfektionen als stilistische Eigenarten im Sinne «rhetorischer> Figuren und Techniken - und somit als zulässige Abweichungsmuster - zu deuten. 
Selbst innerhalb der Werke renommierter Autorinnen und Autoren können mitunter «echte〉 Fehler auftreten, die im Lektorat einzeln beurteilt und allenfalls korrigiert werden müssen. In diesem Prozess kommt die Entscheidung zwischen 〈Fehler〉 und 〈Figur〉 ganz praktisch zur Anwendung. Brisant wird diese Eingriffsmöglichkeit des Verlags als Autorität im postkolonialen Kontext, wie Robert Stockhammer unter Rückgriff auf das Konzept des «Barbarismus〉 - also eigentlich den «barbarischen〉 Fehler - anschaulich aufzeigt. Am Beispiel des Romans «The Palm-Wine Drinkard des nigerianischen Autors Amos Tutuola von 1952, «a novel which is praised for its original use of English - but whose manuscript nevertheless shows the editor's corrections of Tutuola's baddest blunders» (Stockhammer 2005: 303-304, Fn. 1), macht Stockhammer darauf aufmerksam, dass in der Beurteilung und beim Lektorat offenbar eine Trennlinie gezogen wird zwischen Textstellen, die schlicht «krude Fehler» enthalten («baddest blunders») und Stellen, die aus Sicht des Verlags als «originelle` Merkmale innovativer Sprachkunst gedeutet werden («original use of English»). Das Problematische an dieser Unterscheidung ist nun laut Stockhammer, dass sich die korrigierten grammatikalischen 〈Fehler〉 von der 〈rhetorischen Abweichung〉 rein formal überhaupt nicht unterscheiden lassen. Beide sind nämlich durch die Abweichung von der gewöhnlichen, naheliegenden Form definiert, so dass - «the borderline between figura and vitium is hard to control» (Stockhammer 1995: 305). Somit ist jede Unterscheidung von 〈Fehler〉 und 〈Figur〉 durch eine gewisse interpretatorische Willkür geprägt - und hängt somit auch von den Standpunkten und entsprechenden Machtpositionen jener Personen ab, die über 〈richtig〉 und 〈falsch〉 entscheiden. Stockhammer zitiert eine Stelle aus einer Vorlesung des südamerikanischen Schriftstellers J.M. Coetzee, welche über die Problematik dieser Verlagspraxis reflektiert:

The language of Amos Tutuola is English, but not standard English, not the English that Nigerians of the 1950s went to school and college to learn. It is the language of a semieducated clerk, a man with no more than elementary schooling, barely comprehensible to an outsider, fixed up for publication by British editors. Where Tutuola's writing was frankly illiterate they corrected it; what they refrained from correcting was what seemed authentically Nigerian to them, that is to say, what to their ears sounded picturesque, exotic, folkloric. (Coetzee 2003: 46-47; Hervorhebungen A.S.)

Zeugnisse von offensichtlicher Ungebildetheit («frankly illiterate») im Gegensatz $\mathrm{zu}$ authentischer Eigenheit («authentically Nigerian») - oder auch gefälligem Exotismus oder Folklorismus - werden aus einer nicht unproblematischen kolonialistisch geprägten Geschmacksvorliebe und Autoritätsposition heraus unterschieden. Wie aber lässt sich entscheiden, «whether the deviation [...] is due to the author's free choice or to his lack of linguistic skill?» (Stockhammer 2005: 305). 
Stockhammer führt die westliche Distinktionspraxis auf eben jene «Sprachprozeßordnung» (305, in Anlehnung an Walter Benjamins Begriff) zurück, die Quintilian durch die Unterscheidung einer rhetorischen und einer grammatikalischen Ebene erst eingeführt haben soll: Indem Abweichungen entweder dem «Gericht» der Grammatik oder der Rhetorik delegiert werden, lassen sie sich je nach Gutdünken der Auctoritas - als «legitime〉 Kunstmittel verteidigen oder als Fehler verurteilen (Stockhammer 2006: 305). Die Frage nach Fehler oder Figur, Lapsus oder Kalkül, nach «wrong or young», nach Schnitzer oder innovativem Ausdruck stellt sich nicht nur bei Texten im postkolonialen Umfeld, sondern bei allen Werken der Literatur, die innovativ oder experimentell mit Sprache umgehen, die umgangssprachliche Dialoge oder dialektale Färbungen einbringen oder Anakoluth, Asyndeton oder Abbrüche als Stilmittel verwenden. Die Darstellungstechniken des Bewusstseinsstroms oder des inneren Monologs gehen fast notwendig mit Abweichungen von grammatikalischen Regeln einher und auch spontane Dialoge im Sinne von Kleists «allmählicher Verfertigung der Gedanken beim Reden > bedingen eine Anpassung an das freie und entsprechend ungeschliffene Reden. Das unvorbereitete Sprechen, das in der Rhetorik die Stegreifrede charakterisiert, ist aus Sicht der Linguistik gerade gekennzeichnet durch eine «stilistische Unvollkommenheit und vor allem durch eine sfehlerhafte» und damit «brüchige` Syntax» (Antos 1981: 202).

In der heutigen Linguistik werden abweichende Sprachphänomene nicht mehr zwingend als Fehler, sondern als Varietäten der Standardsprache gesehen (z.B. Dialekte, Idiolekte oder Jargon). Dennoch ist uns Quintilians Grenzziehung zwischen Grammatik und Rhetorik so geläufig geworden, dass sie uns bei der Lektüre, Interpretation und Beurteilung von Texten schlicht «unterläuft〉. Liest man eine von der Grammatik abweichende Textstelle, stellt sich dem Leser unweigerlich die Frage: Ist dem Korrektorat ein Lapsus unterlaufen oder muss das so sein? Auch ohne diese Trennung von Grammatik und Rhetorik mit Stockhammer generell in Frage stellen zu müssen, sollten wir uns bewusst sein, dass sie unweigerlich die Problematik der Autorisierungsmacht mit sich bringt, bei der es in letzter Konsequenz um die Zu- oder Aberkennung von Literarizität gehen kann. Umgekehrt bieten aus Sicht der Textproduktion und -rezeption gerade die unscharfen Grenzen zwischen Stilmittel, Fehler und Dilettantismus ein Spannungsfeld, mit dem Autorinnen und Autoren ganz bewusst spielen können und an dem sich Leser, Literaturwissenschaftlerinnen und Kritiker erstaunen, stoßen, erfreuen - oder die Köpfe zerbrechen können. Irritierende Abweichungsmuster bilden eine wichtige Quelle der Inspiration und Wirkkraft literarischer Texte. Während journalistische oder wissenschaftliche Texte eng an die Vorgaben der Grammatik und Verständlichkeit gebunden sind und sich an die in ihrem Kontext vorgegebene, «normale` Form halten müssen, genießt die Literatur die 
〈Freiheit zur Imperfektion〉. Mit dem Wachsen des Renommees oder angesichts des Gesamtwerks einer Autorin oder eines Autors - und durch die Weiterverbreitung abweichender Stilmerkmale - können zunächst irritierende Imperfektionen wieder zur 〈Norm〉 und damit als rhetorische Momente erkennbar werden und entsprechend zum «ungetrübten〉 Lesegenuss gereichen.

\subsubsection{Die Zerstörung der schönen Rede}

Genau solche prozesshaften Veränderungen, Abweichungen, Neukonstellationen und Verschiebungen von Elaboration und Imperfektion beobachtet Renate Lachmann in der Literaturgeschichte. Lachmanns Analyse in «Die Zerstörung der schönen Rede> (1994) ist doppelt erkenntnisreich: Sie zeigt nicht nur die Bewegungen und Umformungen literarischer Stile im Wechselspiel von Regel und Abweichung auf, sondern bringt diese Prozesse mit dem rhetorischen System und seinen immanenten Zyklen von Kritik, Regelbruch, Revision und Erneuerung in Verbindung. Die Triebfeder für diese Entwicklungen schließlich verortet sie, in ganz ähnlicher Weise wie diese Arbeit, in der immerwährenden Suche nach einer authentischen Ausdrucksform.

Ähnlich wie bei Stockhammer führt die Rhetorik auch bei Lachmann zur «Etablierung eines Kodes zulässiger Uneigentlichkeit» (Lachmann 1994: 13), welcher bestimmte Fehler und Abweichungen als Stilmittel lizenzieren kann. Dabei unterscheidet sie jedoch zwischen einer konservativen und einer progressiv angelegten Rhetorik. Während die regelbestätigende oder Decorum-basierte Rhetorik ein fixes Regelinventar herausgibt, das angemessene Stile, «Metaphernschätze» und «Argumentenrepertoires» für unterschiedliche Gegebenheiten bereithält (Lachmann 1994: 16), ist ihr «Gegentyp [...] eher innovativ, d.h. es kommt zur Formulierung neuer Regeln, die als Verstöße gegen die bestehenden verstanden werden können. (Oder anders: Die Verstöße werden zu Regeln erhoben). Dieser Typ kann eine antizipatorische (oder avantgardistische) Aufgabe übernehmen.» (Lachmann 1994: 17). Historisch führt Lachmann den aus den Ästhetikkontroversen des 18. Jahrhunderts erfolgten Bruch mit der Rhetorik auf das damals vorherrschende, regelverhaftete Rhetorikverständnis zurück, welches für eine «Neuerung von innen nicht offen gewesen sei. Die komplette Abkehr von der Rhetorik habe letztlich zu einer begrifflichen Abspaltung des 〈Rhetorischen〉 vom 〈Poetischen〉 geführt, wobei das «Rhetorische〉 fortan schlechthin als regelgebunden, unkreativ und nicht spontan gegolten habe, während das «Poetische» für Regelfreiheit, Spontaneität, für das Schöpferische und dieses «unterstützende Vorstellungen wie Originalität, Einbildungskraft, Inspiration und Genialität» gestanden habe (Lachmann 1994: 17). Erst im Strukturalismus und Formalismus des 20. Jahr- 
hunderts sei, so Lachmann, wieder deutlich geworden, «daß Texte auch nach der Verwerfung der Rhetorik mit Verfahren aufgebaut werden, die mithilfe der Rhetorik definiert werden können. D.h. auch der scheinbar regelenthobene Text funktioniert rhetorisch.» (Lachmann 1994: 19).

In den Phasen der Ablehnung oder Unsicherheit gegenüber einem dominanten System können nun nach Lachmann «Tendenzen relevant werden, die gegen diese systemstützende Rhetorik gerichtet sind oder sich unabhängig von ihr herausgebildet haben.» (Lachmann 1994: 9). Einerseits die «Volkspoesie, die sich nicht an einer kodifizierten Rhetorik orientiert», andererseits Fälle «einer kommunikativen (poetischen) Praxis [...], die bewußt gegen ein herrschendes rhetorisches Reglement gerichtet ist und diesem gegenüber eine Anti- oder Subrhetorik entwickelt, die Sprachebenen einbezieht, die die offizielle Rhetorik gerade nicht zuläßt.» (Lachmann 1994: 9-10). Diese subversiven Tendenzen weisen nicht nur Parallelen auf $\mathrm{zu}$ jenen gestalterischen 〈Antihaltungen〉 und dem Zelebrieren dilettantischer Formen, wie wir sie im Grafikdesign des Punk oder der Postmoderne kennengelernt haben, sondern lassen sich, wie Lachmann überzeugend ausführt, auch auf Entwicklungen in der Literatur anwenden. Am Beispiel der literarischen Situation in der russischen Literatur des 18. und 19. Jahrhunderts zeigt sie auf, wie literarische Stil- und Elaborationsformen historisch im Zusammenhang mit Prozessen der Erneuerung, Konsolidierung und Wiederablösung, der Bewegung und Gegenbewegung zu begreifen sind, die letztlich von der Suche nach Authentizität angetrieben werden. So komme der Sentimentalismus in der russischen Literatur just in jener Zeit auf, in welcher sich die rhetorischen Regelpoetiken der Renaissance und des Barock zum «Inbegriff einer Stilmanier der Phrasenhaftigkeit, Unaufrichtigkeit, Trivialität und ästhetischen Erschöpftheit» verflacht hätten (Lachmann 1994: 284). Diesem starr gewordenen Regelwerk werde mit dem Sentimentalismus eine personalisierte Form des Dichtens und des unverfälschten Gefühls entgegengesetzt, manifestiert in einem «empfindsamen Selbst», sowie einem neuen, «angenehmen», mittleren Stil (Lachmann 1994: 284).

Sobald die Sentimentalisierung der Literatur jedoch $\mathrm{zu}$ einem Stil vereinheitlicht und von weiteren Autoren übernommen wird, verfestigt sich dieser laut Lachmann erneut «zu einem manierierten Habitus, und eine empfindsame, den gesamten Prozeß der Bedeutungskonstitution lenkende Perspektive wird zum Diktat.» (Lachmann 1994: 286). Der empfindsame Dichter verkomme dabei zur «Schreibermaske» und die «durch persönliche Autorschaft verbürgte «Aufrichtigkeit» der Weltwahrnehmung» verliere ihre «appellative Funktion» (Lachmann 1994: 286-287). Sowohl die Stilqualität des «Angenehmen〉 als auch die Personalisierung der Literatur wird gemäß Lachmann nur so lange als «Innovation» wahrgenommen, als sie «das Versprechen einer neuen Sensibilität und bislang ungekannten Unmittelbarkeit und Authentizität des Ausdrucks einlösen» kann 
(Lachmann 1994: 286). Aus der Stereotypisierung, Automatisierung und Trivialisierung des vermeintlich «authentischen` oder «ungekünstelten` Ausdrucks heraus komme deshalb in der russischen Literatur ein neuer «Rhetorismus >-Vorwurf auf, der schließlich in den Realismus überleite: «an die Stelle der kompromittierten Realität des Gefühls tritt die Realität der sozialen Wirklichkeit» (Lachmann 1994: 287). Auch innerhalb des Realismus stellt Lachmann wiederum Bewegungen der Konsolidierung und Infragestellung fest, denn gerade durch den Anspruch an die Wiedergabe der Realität rücke zugleich auch die «Problematik der «darstellenden Mittel»» in den Mittelpunkt (Lachmann 1994: 287).

Werde in einer ersten Phase noch aufrichtig versucht, durch eine Reduktion der Stilmittel und durch «immer neue Wege der Entmedialisierung» (Lachmann 1994: 288) 〈unverfälschte> Formen der Wirklichkeitsvermittlung zu erlangen, münde dieses Streben mehr und mehr in die Deformation und Persiflage der Realitätswiedergabe, welche die Unmöglichkeit des Vorhabens einer «Annäherung an das Leben» (Lachmann 1994: 288) freilege. So stelle Alexander Puschkin der Schwülstigkeit, den «welken Metaphern» und dem bloßen Wohlklang der «schönen Rede» des Sentimentalismus noch «Qualitäten wie Konzisheit, Präzision, vor allem aber Prägnanz, die als positive stilistische Umsetzungen seiner kritischen Einstellung gegenüber Formelhaftigkeit, Trivialität und Redundanz erscheinen», entgegen (Lachmann 1994: 291). Da Puschkin gleichzeitig jede Form von «Stil` im Widerspruch zum «Realen` sehe, gehe es ihm dabei ganz eigentlich um einen «Verzicht auf Stil», weshalb er in seinen Texten denn auch auf Elemente der «Volkssprache» zurückgreife, die «als unterste Schicht der im 18. Jahrhundert aufgestellten Sprachhierarchie von einer Rhetorisierung» unangetastet geblieben sei (Lachmann 1994: 292). Sobald sich Puschkins «Anti-Stil〉 in Form der sogenannten «Natürlichen Schule` jedoch etabliert hat und seine Innovationen kanonisiert sind, führt dies, so Lachmann, wiederum zu einer «Entaktualisierung», bei welcher der «provokative Effekt» und die Aggressivität der Puschkinschen Prosa verloren gehe (Lachmann 1994: 293).

In einer nächsten Phase radikalisiert sich das Realismusprogramm laut Lachmann weiter und entwickelt - über Puschkins «Gegenstil` hinaus - einen neuen Gattungstyp: die Skizze: «In der Skizze tritt das narrative Moment zugunsten eines deskriptiven, das dem Objekt, dem Milieu gilt, zurück.» (Lachmann 1994: 297-298). In Lachmanns Darstellung vollzieht Nikolai Gogol als letzte Stufe der Anti-Kunst - die zugleich zur Auflösung des Realismus von innen führt - schließlich den Schritt zum «naturalen, pointiert unliterarischen Sprechen nicht nur auf Ebene der Protagonisten, sondern auch auf der der Erzähler» (Lachmann 1994: 298). In Abweisung des auktorialen Erzählers entwickle er «eine Art Antierzähler, der seine Unfähigkeit, mit den Stereotypen der schönen Erzählrede umzugehen, sprachlich «zur Schau stellt» (Lachmann 1994: 298). Im Gegensatz zur 
Sprache der Skizzen sei Gogols Sprache bereits «alles andere als natürlich» und seine Figurendarstellung nunmehr eine «Scheinbeschreibung» (298). Lachmann stellt entsprechend die These auf, dass Gogol die Gefahr der Verfestigung und Schemabildung der realistischen Darstellung habe kommen sehen, weshalb er in parodistischer Weise auf das vermeintlich «natürliche` Sprechen eingegangen sei. Durch den Rekurs auf Anakoluth, Lapsus oder «den demonstrative[n] Verzicht auf die Differenzierung von Stilebenen» versuche Gogol nur scheinbar einen Eindruck von normalsprachlicher, mündlicher Rede zu erzeugen - tatsächlich jedoch kreiere er in spielerischer Weise und durch unmotiviertes Vermischen von Stilhöhen eine «uneigentliche Mündlichkeit» und somit eine «hochkomplexe, künstliche Stilqualität» (Lachmann 1994: 299). Damit führt Gogol gemäß Lachmann die «Stilkritik im Blick auf eine wirklichkeitsadäquate Darstellung» ganz eigentlich «ad absurdum» (299).

Formen, die also von den Skizzenschreibern noch «seriös» gebraucht wurden, werden bei Gogol bereits «ludistisch» eingesetzt (Lachmann 1994: 300). In der radikalsten Form dieses «defekten Stils» überlasse Gogol seine Protagonisten gar der Aphasie: «Stottern und Verstummen, die Reihung von Partikeln und Satzfetzen lassen in der Deformation der Sprache und der unschönen Rede die Stilreflexion eskalieren.» (Lachmann 1994: 300). Sobald mit Gogol die äußerste Möglichkeit des Anti-Stils beschritten sei, bleibe Fjodor Dostojewski nur der Sprung auf die Metaebene. Indem er seine Figuren unterschiedlich sprechen lasse, habe sich Dostojewski sowohl den phrasenhaft unauthentischen als auch den defekten Stil angeeignet und diese bewusst aufeinanderprallen lassen, was von Lachmann wiederum als versteckter poetologischer Kommentar zur geführten Debatte interpretiert wird: «Weder die verstummte und defekte, noch die beredte, aber verfälschende Sprache kann 〈reale Funktionen〉 in der Wirklichkeitsvermittlung übernehmen. Indem sie der einen entgleitet, wird sie von der anderen usurpiert.» (Lachmann 1994: 302).

Mit diesem Suchen, Winden und Überwinden literarischer Elaborationsund Darstellungsverfahren ist nichts anderes beschrieben als das immer wieder neue, aber im Grunde unmögliche Bestreben, Echtheit und Unmittelbarkeit in der Kunst zu erlangen. In jenem Moment, in dem das Paradox der Authentizität erkannt wird, erfolgt der Bruch und wird in einer eigentlichen Umkehrung der Dissimulatio Artis die Vermitteltheit der Kunst, ihre Künstlichkeit zur Schau gestellt - bis wieder ein Versuch in eine neue Richtung unternommen wird. 


\subsection{Schluss}

Was Renate Lachmann unter dem Aspekt des 〈Rhetorismus $\rangle$-Vorwurfs für die russische Literatur des 18. und 19. Jahrhunderts aufzeigt, weist so frappante Parallelen zum Zusammenprallen von Elaborationsideal und Imperfektion und den daraus resultierenden Entwicklungslinien auf, die in dieser Arbeit - ebenfalls mit Blick auf die Rhetorik - im Grafikdesign des 20. und 21. Jahrhunderts nachgezogen wurden, dass man in diesem Wechselspiel nicht nur einen Grundzug der Künste oder Téchnai, sondern fast schon eine anthropologische Konstante vermuten könnte. Lassen sich ähnliche Bewegungen und Gegenbewegungen vielleicht auch in weiteren literarischen Stilrichtungen und Strömungen, in anderen Designbereichen und Künsten, zu anderen Zeiten und in weiteren Kulturräumen beobachten?

Bleiben wir beim Dreieck von Literatur, Rhetorik und Design, so erinnert die von Puschkin geforderte Konzisheit, Präzision und Prägnanz an die Elaborationsprinzipien der Reduktion, Schlichtheit und Akribie, die wir bereits aus der klassischen Rhetorik, aber auch aus dem modernistischen Design kennen. In analoger Weise verbindet er damit zudem eine Kritik an übermäßiger Ausschmückung oder abgeschmackter Metaphorik. Auch Puschkins Rückgriff auf die 〈Volkssprache〉, die bewusste Verwendung einer unvollendeten, flüchtigen Elaborationstechnik in der Skizzenliteratur und selbst die komplette Zerstörung und Auflösung des Stils bei Gogol sowie die Metabetrachtungen und Stilbrüche von Dostojewski haben wir in ähnlicher Weise in der Grafik des Dada, des Punk, der Postmoderne oder des «Handmade〉 kennengelernt. Sie alle entfalten ihre Sprengkraft aus der Imperfektion und aus dem Regelbruch - können ihre Wirkkraft als Gegenbewegungen jedoch nur für beschränkte Zeit entfalten. Puschkins (vermeintlicher) 〈Stilverzicht〉, mit dem er Authentizität erreichen wollte, gemahnt überdeutlich an das provokante Verweigerungsvotum 〈Style = FART 〉 des Grafikdesigners Stefan Sagmeister von 1999, weshalb ich hier mit einem kurzen Auszug aus einem Interview mit Sagmeister enden möchte, das gut zehn Jahre nach seiner Provokation im Magazin der «Süddeutschen Zeitung〉 erschienen ist:

Von Ihnen stammt der Spruch: Stil ist Furz. Zumindest haben Sie sich das mal für ein Plakat in Ihren Bauch ritzen lassen. Zählt also allein die Idee?

Das war ein Fehler. So habe ich vor ewigen Zeiten gedacht: dass Stil an sich unwichtig ist, eben nur heiße Luft. Das sehe ich heute überhaupt nicht mehr so.

Es gibt ja auch eindeutig einen Sagmeister-Stil: plakativ, bunt, provozierend.

Das habe ich erst spät zugelassen: dass es eine formale Richtung geben kann, die wiedererkennbar wird. Vorher hatte ich die fixe Idee, unser Studio müsse sich immer wieder neu erfinden. Und das hat sich sehr flott als absolut unmöglich herausgestellt. Es war irgendwann klar, dass das nur möglich ist, wenn ich entweder historische Stile stehle oder noch 
schlimmer: Stile von anderen Designern übernehme. Für jedes Projekt einen neuen Stil zu erfinden, das hat noch keiner geschafft. (Bärnthaler 2011)

Wie in diesem Buch gezeigt wurde, geht es im Design ebenso wie in der Rhetorik und in der Literatur immer wieder um die letztlich unauflösbaren Widersprüche von Kunst und Natürlichkeit, Verfeinerung und Rohheit, Akribie und Lockerheit, Genauigkeit und Spontaneität, Manipulation und Aufrichtigkeit, Seriosität und Vertrautheit, Konvention und Provokation, Wiedererkennbarkeit und Neuheit, Anpassung und Abweichung, Verfälschung und Echtheit. Dies manifestiert sich im spannungsvollen Wechselspiel, in der Dialektik von Elaboration und Imperfektion, von Geschliffenheit und Ungeschliffenheit, von perfekt und unperfekt. 
